\title{
Le développement historique du discours sur la qualité de la population au Japon
}

The Historical Development of the Discourse on Population Quality in Japan

\section{Homei Aya}

Traducteur : Traduction Isabelle Konuma

\section{(2) OpenEdition \\ Journals}

\section{Édition électronique}

URL : https://journals.openedition.org/cipango/4468

DOI : 10.4000/cipango.4468

ISSN : 2260-7706

\section{Éditeur}

INALCO

Édition imprimée

Date de publication : 20 octobre 2021

ISBN : 9782858313969

ISSN : $1164-5857$

Référence électronique

Homei Aya, «Le développement historique du discours sur la qualité de la population au Japon », Cipango [En ligne], 24 | 2021, mis en ligne le 15 octobre 2021, consulté le 08 avril 2022. URL : http:// journals.openedition.org/cipango/4468 ; DOI : https://doi.org/10.4000/cipango.4468

\section{(c) (i) (9)}

Cipango est mis à disposition selon les termes de la Licence Creative Commons Attribution - Pas d'Utilisation Commerciale 4.0 International. 


\title{
Le développement historique du discours sur la qualité de la population au Japon
}

\author{
The Historical Development of the Discourse \\ on Population Quality in Japan
}

Homei Aya

Lecture at University of Manchester

Traduction Isabelle KonUma

$$
\text { Inalco - IFRAE }
$$

Il est indéniable que la qualité s'exerce toujours sur quelque forme que ce soit. 形のあるところ、絶えず質がこれを左右

していることは否定できない。

Shinozaki Nobuo 篠崎信男, octobre $1979^{1}$

\section{Introduction}

En histoire du Japon moderne, l'eugénisme et l'hygiène raciale furent longtemps considérés comme ayant conforté le concept de race (minzoku 民族 ou jinshu人 種), ce qui aurait permis l'avènement d'une nation moderne, autrement dit d'un

1. Shinozaki, octobre 1979, p. 26. 
empire $^{2}$. En croisant différentes perspectives telles que les mouvements sociaux, la science, l'industrie et la politique étatique, ces études mirent en exergue la façon dont l'avortement, la stérilisation, le contrôle des naissances et toute autre pratique reproductive furent effectués au Japon et dans ses colonies avant, durant et après la guerre d'Asie-Pacifique ${ }^{3}$, avec la participation des experts médico-scientifiques eugénistes ${ }^{4}$.

Les considérations eugénistes agirent continuellement sur les débats politiques des années 1910 aux années $1960^{5}$. Pourtant, cette perspective a des limites car, en se focalisant sur l'eugénisme, on se prive d'une vue plus large sur les politiques de population en général. Comme l'a récemment démontré Sugita Naho 杉田菜 穂, spécialiste de la politique sociale, l'euthénisme ${ }^{6}$, qui cherche à élever le niveau de vie par des mesures sociales et de bien-être, aurait façonné les politiques de population de la même manière que les politiques eugénistes ${ }^{7}$. Il devient alors crucial de croiser une approche essentiellement biologique de la population telle que le sang et la génétique (eugénisme) avec une compréhension plus large de l'amélioration générale du bien-être de l'homme (euthénisme). Ce prisme est particulièrement significatif puisqu'il nous permet de saisir les continuités historiques qui ont caractérisé la gouvernance de la population au Japon avant et après la guerre ${ }^{8}$.

2. Schaffner, 2014 ; Yamazaki, 2013 ; Robertson, 2010 ; Fujino, 1998 ; Chung, 2002 ; Оtsubo, 1999 ; Matsubara, 1998.

3. Ogino, 2008a ; Tama, 2006 ; Ōbayashi, 2006 ; Norgren, 2001 ; Tipton, 1994.

4. Matsubara, 2002, p. 104-106; Matsubara, 2000 ; Oguma, 1994 ; Hiroshima, $1980 \& 1981$.

5. Les « politiques de population» (jinkō seisaku 人口政策) furent expressément mises en place durant la guerre. Toutefois, les débats et propositions politiques qui les précédèrent sont tout aussi importants en cela qu'ils devinrent les fondations des politiques de population durant la guerre. Voir SU Gita, 2010.

6. Le mot fut traduit par yūkyōgaku優境学 par le sociologue Takebe Tongo 建部挀吾 (1871-1945).

7. Sugita, 2013.

8. La question de la continuité historique n'a pas été posée uniquement par Sugita mais aussi par Matsubara et Ogino Miho : Sugita, 2010, p. 199-207 ; Ogino, 2008b ; Matsubara, 1998. 
Durant l'après-guerre, la reprise du concept de « développement » (kaihatsu開 発) est un élément à ne pas négliger. Le développement était certes déjà un sujet de débats avant la guerre, essentiellement en lien avec le développement économique et sanitaire de la région du Tōhoku' ${ }^{9}$. Toutefois, dans la période d'après-guerre, avec l'adoption du néologisme 《développement social 》 (shakai kaihatsu 社会 開発), la sémantique change, le « développement » couvre désormais un sens plus large teinté de la théorie de modernisation ${ }^{10}$.

L'importance $\mathrm{du}$ 《développement »dans la politique intérieure fut déjà avancée par Sugita et Matsubara ${ }^{11}$. Tout en intégrant ces études, nous avançons ici que la notion de développement permit d'établir un lien entre la politique intérieure du Japon et la politique mondiale de population. Afin d'aborder cette question, nous nous pencherons sur la concomitance entre l'émergence du développement social dans les débats intérieurs et la participation du gouvernement japonais au mouvement de planning familial transnational visant à contrôler le surplus démographique dans les « pays en voie de développement » (hatten tojökoku 発展途上国) par le biais des aides au développement ${ }^{12}$. Nous soutenons que cela n'était ni une coïncidence ni un accident : des efforts furent entrepris avec détermination puisque le Japon aspirait alors à rejoindre, en tant que pays 《semi-développé » (chüshinkoku 中進国), les membres des « pays développés 》(hattenkoku 発展国) ${ }^{13}$. En intégrant ces différents points, le présent article propose de tracer la généalogie du concept de qualité de la population dans les débats politiques depuis les années 1910 jusqu'aux années 1960, en cherchant à éviter le cloisonnement lexical qui empêche de saisir l'existence d'une politique globale sur l'amélioration de la qualité de l'homme.

9. Hopson, 2017.

10. ЕSCOBAR, 1995.

11. Sugita, 2015a; Matsubara, 2000, p. 192-194.

12. McCann, 2016 ; Latham, 2011 ; Connelly, 2008 ; Donaldson, 1990.

13. Номеi, 2016. 
CIPANGO

98 Cahiers d'études japonaises nº 24

\section{Avant 1945 : entre eugénisme et euthénisme}

Dans la période d'avant-guerre au Japon, l'eugénisme, interprété dans un sens étroit en lien avec la génétique mendélienne, constitua l'un des piliers idéologiques de l'amélioration de la qualité de la population, ou plus précisément de la « race japonaise ${ }^{14} \gg$.Le débatsurl'eugénisme pénétra parmiles médecinsetles scientifiques durant le début du $\mathrm{Xx}^{\mathrm{e}}$ siècle, le Japon étant alors en pleine construction d'une nation forte afin de survivre face à la compétition internationale. Les eugénistes étaient alors divisés entre deux grands courants de pensée concurrents. Le premier courant, diffusé par les intellectuels tels que Unno Yukinori 海野幸徳, auteur du livre De l'amélioration de la race japonaise (Nihon jinshu kairyōron 日本人種 改良論) en 1910, encourageait le métissage pour renforcer la qualité de la race japonaise. Cette position, explicitée par l'expression “mixed blood” (zakkon 杂低婚) theory, soutenait les bienfaits du métissage entre différents groupes raciaux ${ }^{15}$.

Le second courant soutenait quant à lui la "pure blood" (junketsu純血) theory. Cette vision était celle de chercheurs tels que Nagai Hisomu 永井潜, appartenant à l'université impériale de Tōkyō et à la tête du mouvement eugéniste avant et durant la guerre ${ }^{16}$. Les chercheurs de ce courant s'opposèrent au métissage au motif que la race inférieure reproduisait ses mauvais traits au sein de la race supérieure, provoquant ainsi la dégradation de la qualité de cette dernière.

Ces deux courants furent présents tout au long de la période précédant et durant la guerre, avec une intensité variable. De façon générale, la théorie du « sang métissé », minoritaire chez les eugénistes, servit de fondement aux mesures d'assimilation coloniale pour souligner le bénéfice que l'on pouvait tirer de l'unité entre les Japonais et les sujets coloniaux ${ }^{17}$. En revanche, la théorie du «sang pur »

14. Sur l'histoire de l'eugénisme, voir BASHFORD \& LEVINE, 2010. Spécifiquement pour l'histoire moderne de l'eugénisme au Japon, voir SCHAfFner, 2014 ; FujIno, 1998 ; Suzuki, 1983.

15. Robertson, 2010 ; Oguma, 1994.

16. Sur le travail de Nagai, voir Chūman, 2013 ; Chung, 2002 \& 2013 ; Fujıno, 1998, p. 56-60 \& 2003, p. 30 ; OGUMA, 1994, p. 152-157\& 1995.

17. Weiner, 1994. Voir l'article d'Arnaud Nanta dans le présent numéro. 
devint progressivement dominante dans le processus d'élaboration des politiques de population durant la guerre ${ }^{18}$.

Les années 1920 connurent une poussée de débats sur la qualité de la population en lien avec le problème de « surpopulation » (kajō jinkō 過剩人口) rendu visible par les premiers recensements nationaux de 1920. Sous l'influence de la théorie malthusienne, les écrits officiels s'inquiétèrent de l'impact imminent de la «surpopulation » dans le domaine de la distribution alimentaire. Par conséquent, en 1927, le gouvernement mit en place la Commission d'enquête sur la population et le ravitaillement (Jinkō shokuryō mondai chōsakai 人口食糧問 題調査会, ci-dessous CEPR) au sein du Cabinet. Elle fut la première et unique instance compétente en matière d'enquête et de délibération auprès des ministères sur les questions de population ${ }^{19}$.

La CEPR fut alors chargée de formuler un projet sur le contrôle de la population, avec Nagai Hisomu en son centre ${ }^{20}$. C'est ainsi que l'eugénisme fut hissé parmi les principales mesures débattues au sein de la CEPR. Les Diverses mesures concernant le contrôle de la population (Jinkō tōsei ni kansuru shohōsaku 人口統制二関スル諸方策), l'une des propositions phares de cette instance soumise au gouvernement en 1929, interprétaient le contrôle de la population en ces termes :

Il n'est pas question d'agir uniquement sur le nombre mais aussi sur la qualité d'un point de vue eugénique. [...] Les mesures de contrôle de la population sont l'ensemble des mesures cherchant à rendre la population saine tant sur le plan de la quantité que sur le plan de la qualité.

単二人口数ノ問題ノミナラズ、優生学的見地ヨリスル 人口ノ質ノ向上ヨモ意図スルモノナリ。 …数及質ノ上

18. OGuma, 1994.

19. Jinkō ShokURyō MONDAi ChōSAKaI, 1930, p. 1. Pour plus de détails, voir DinMORE, 2006, p. 14-58.

20. Professeur à l'université impériale de Tōkyō, il fut à la tête du mouvement eugéniste avant et durant la guerre. 


\section{二於テ健全ナル人ロ状態习実現セシメントスル方策ヨ一 括シテ人口統制二関スル諸方策卜謂ヒタルモノナリ。 21}

Trois recommandations sur neuf portaient sur des principes eugénistes : « mettre en place des établissements adaptés pour dispenser des consultations médicales sur le mariage, l'accouchement et la contraception » (mesure 7); « renforcer le contrôle des infractions en relation avec la diffusion, la vente et la publicité des articles et médicaments contraceptifs » (mesure 8) ; « mener des recherches sur différentes institutions du point de vue eugénique » (mesure 9).

Pour comprendre la portée eugéniste de la huitième mesure, il faut saisir l'impact supposé néfaste de la baisse de la natalitée ${ }^{2}$. En effet, les eugénistes étaient particulièrement méfiants envers la natalité, dont la baisse était le signe de la propagation du contrôle des naissances parmi les classes intellectuelles de la métropole ${ }^{23}$. Cette crainte eut un impact important au sein du camp des eugénistes favorables au « sang pur » représentés par Nagai Hisomu, pour qui la race japonaise perdait de sa qualité en raison de la « dégénérescence » (gyaku tōta 逆淘汰). Ce dernier concept fut défini par Koya Yoshio 古屋芳雄, hygiéniste et collègue de Nagai, comme étant « le processus par lequel les individus de qualité avec des traits robustes ont de moins en moins de descendants pendant que les individus inférieurs et faibles se propagent »優良強健な分子が次第にその 子孫を喪失し却つて不良劣弱な分子が増殖して行くことをいふので ある24.

Les débats tenus devant la CEPR sont fascinants en cela qu'ils montrent précisément comment une autre idéologie que la pensée eugéniste put venir renforcer les débats sur la qualité de la population. Parmi ces idéologies se trouve l'euthénisme qui se traduit par la promotion du bien-être de l'homme à l'aide de l'amélioration des conditions entourant la vie en général. La figure centrale

21. JiNKŌ SHOKURYŌ MONDAI CHŌSAKAI, 1930, p. 118. Concernant les débats autour de cette proposition, voir Sugita, 2010, p. 190-199; Hiroshima, 1980.

22. Si la population continuait à augmenter, le taux de naissance connaissait quant à lui une baisse, passant de 361,95 pour 10.000 personnes en 1920 à 323,52 en 1930 .

23. JINKŌ SHOKURYŌ MONDAI CHŌSAKAI, 1930, p. 120.

24. Koya, 1941, p. 40. 
fut Nagai Tōru永井亨, l'un des experts sociaux les plus influents entre les années 1920 et les années $1960^{25}$. Tout comme Hisomu ${ }^{26}$, Tōru faisait partie des conseillers en matière de population auprès du gouvernement et il était l'un des principaux membres de la CEPR. Or, il se démarquait de l'eugénisme de Hisomu puisqu'il avançait une alternative à la promotion de la qualité de la population, prenant appui sur l'euthénisme ${ }^{27}$.

Tōru interprétait la qualité de la population en termes d'équilibre démographique (âge, sexe, etc.). Sans pour autant rejeter l'eugénisme, il prônait les bienfaits des 《établissements sociaux 》(shakaiteki shisetsu 社会的施設), en particulier ceux destinés aux mères et aux enfants ${ }^{28}$. Les Diverses mesures concernant le contrôle de la population intégrèrent d'ailleurs cette dimension et préconisèrent des « exercices physiques » ainsi que la «promotion de l'état nutritif chez les filles » (mesure 3), l'encadrement du travail féminin sur le plan de la santé et de l'hygiène (mesure 4), et la création d'établissements sociaux destinés au grand public sur la protection de la maternité et le soin aux enfants (mesure 6) ${ }^{29}$.

Au cours des années 1930, les tentatives de Tōru menèrent à l'élaboration de nombreuses mesures politiques sur le bien-être de l'enfant et, plus largement, sur le bien-être de la femme ${ }^{30}$. Cependant, son idée fut progressivement évincée par l'idéologie eugéniste du « sang pur ». Les raisons étaient nombreuses, mais le changement institutionnel en était le facteur déterminant. Le 10 avril 1930, on mit fin aux activités de la CEPR dans laquelle Tōru exerçait son pouvoir. Par la suite, il tenta d'œuvrer à travers la Fondation pour la recherche en matière de problèmes de population (Zaidan hōjin jinkō mondai kenkyūkai 財団法人人口問題研究 会, ci-dessous FRPP), mise en place au sein du ministère de l'Intérieur en 1933. Toutefois, avec la création en 1938 du ministère de la Santé, du Travail et des

25. Sur Nagai Tōru, voir Sugita, 2010, p. 184-233 \& 2013, p. 117-141, p. 175-182 \& p. 190-202.

26. Afin d'éviter toute confusion, nous utiliserons exceptionnellement les prénoms (Hisomu, Tōru) pour distinguer les deux personnages partageant le même nom.

27. Sugita, 2010, p. 192-199.

28. Ibid., p. 193.

29. JINKŌ SHOKURYŌ MONDAI CHŌSAKAI, 1930, p. 41-44.

30. Sugita, 2010. 
102 Cahiers d'études japonaises nº 24

Affaires sociales (Kōseishō 厚生省, ci-dessous MSTAS) ainsi que l'établissement en son sein de l'Institut de recherche sur la population (Jinkō mondai kenkyūjo 人口問題研究所, ci-dessous IRP), l'influence de Tōru fut temporairement affaiblie.

Ces changements institutionnels eurent un impact positif chez les partisans de la théorie eugéniste du « sang pur » tels que Hisomu et Koya Yoshio, ce dernier étant étroitement lié au MSTAS en tant qu'expert, et c'est dans ce contexte que la Loi nationale eugénique de 1940 (Kokumin yūseihō 国民優生法) ainsi que le Plan pour l'établissement d'une politique démographique de 1941 (Jinkō seisaku kakuritsu yōkō 人口政策確立要綱) virent le jour. En vertu de la théorie du « sang pur », ces politiques de population partaient de l'idée que les Japonais formaient une population entièrement indépendante et une catégorie raciale à part. Des moyens eugénistes devaient être mis en place pour protéger la qualité de la population, telles que sanctionner le contrôle des naissances et améliorer la capacité physique des individus dotés de traits «supérieurs ». Ces politiques de santé reposaient sur la croyance dans le patrimoine biologique de chaque individu.

Si cet eugénisme ne fut prédominant que pendant une très courte période, entre 1941 et la défaite de 1945, et dans un cercle restreint notamment au sein du MSTAS $^{31}$, cela fut suffisant pour marginaliser la vision de Tōru qui, malgré cela, continua à influencer les politiques sociales.

\section{L'immédiat après-guerre : rupture et continuité}

Au lendemain de la défaite du Japon en août 1945, les débats sur la population refirent immédiatement surface. Comme dans les années 1920, le problème principal était la surpopulation, à la différence que celle-ci était alors causée par une poussée de natalité et l'afflux des rapatriés suite à la perte des colonies. Ces défis spécifiques à la période d'après-guerre se traduisirent par une augmentation démographique de huit millions d'hommes. Combinée avec une insuffisance alimentaire, cette poussée démographique fut rapidement assimilée à une crise

31. En effet, ce courant ne put gagner toutes les sphères des politiques raciales sous l'Empire du Japon, en particulier face aux politiques coloniales d'assimilation : voir l'article d'Arnaud Nanta dans le présent numéro. 
malthusienne ${ }^{32}$. Émergea alors l'image d'un Japon pauvre en ressources avec un futur socioéconomique sinistre, en proie à la famine, au chômage et aux troubles sociaux ${ }^{33}$.

Par ailleurs, sous l'occupation des Alliés (1945-1952), la population devint rapidement un problème politique majeur lié à l'indépendance nationale ${ }^{34}$. L'état de surpopulation symbolisait en particulier une dépendance alimentaire permanente envers les États-Unis, entravant le travail des Alliés qui cherchaient à démilitariser le Japon et à le doter d'un régime démocratique souverain. Par conséquent, la surpopulation devint rapidement un sujet politique majeur chez les Alliés ${ }^{35}$.

Dans ce contexte, le SCAP (Supreme Commander for the Allied Powers) et le gouvernement japonais collaborèrent pour redéfinir les infrastructures compétentes en la matière ${ }^{36}$. Le GHQ (General Headquarters) chargea en premier le MSTAS de cette mission. En mai 1946, le gouvernement relança l'IRP à l'intérieur du MSTAS et somma la FRPP de reprendre ses activités. En avril 1946, la FRPP mit en place le Comité sur les mesures de population (Jinkō seisaku iinkai 人口政策委員, ci-dessous CMP) qui devint un groupe d'experts en politique. Enfin, en 1949, le Cabinet mit en place le Conseil consultatif sur les problèmes de population (Jinkō mondai shingikai 人口問題審議会, ci-dessous CCPP).

D'emblée, les trois organismes - FRPP, CMP et CCPP - tissèrent des liens de collaboration : la FRPP menait des recherches et générait des données sur la population tandis que le CMP, se référant auxdites données, initiait des discussions et soumettait des projets au CCPP qui remettait quant à lui au gouvernement des résolutions écrites. Cette « trinité » fut ainsi dotée d'une immense autorité en matière de politique de population jusqu'à la fermeture de ces instances dans les

32. Sur la crise alimentaire d'après-guerre au Japon, voir FUCHS, 2007.

33. Sur l'impact de la surpopulation sur l'économie, voir O’BRYAN, 2009, p. 146-148.

34. Dinmore, 2006, p. 111-159.

35. Ogino, 2008b, p. 142-151; OAKLEY, 1978.

36. Cependant, du côté des États-Unis, la collaboration se fit discrètement, tant le contrôle des naissances était en soi problématique sur leur propre territoire. 
104 Cahiers d'études japonaises nº 24

années $1970^{37}$. Ainsi, entre les années 1940 et le début des années 1970, ce trio fut à l'origine d'un certain nombre de mesures politiques gouvernementales. Dans ce cadre, le contrôle des naissances, jusque-là évité car associé au socialisme et à la « dégénérescence », fut redéfini comme un moyen de contrôle de la population.

La qualité de la population devint alors un sujet central. La visibilité croissante des orphelins sans famille, des soldats rapatriés sans travail, des vagabonds au chômage, de jeunes Japonaises appelées pan-pan girls associées à des prostituées des GIs américains, et des enfants métisses résultant de ces relations générèrent une anxiété croissante au sein des officiers du MSTAS qui y virent la preuve de la dégénérescence raciale ${ }^{38}$. Entre 1945 et 1946, Ashida Hitoshi, alors ministre de la Santé, du Travail et des Affaires sociales, aurait annoncé que le Japon était confronté à une « crise raciale 》 (minzokuteki kiki 民族的危機) et appela à tenir un nouvel agenda politique pour permettre la « reconstruction de la race [japonaise] » (minzoku fukkō 民族復興) ${ }^{39}$. Suivant les propos d'Ashida, en janvier 1946, le MSTAS organisa un cercle de discussion dans lequel la « promotion de la qualité de la population » fut définie comme une politique universelle de population (jinkō no shitsuteki kōjō wa fuben no jinkō seisaku 人口の質的向上は普遍の人 口政策). Partant de cette idée, le gouvernement japonais fut sommé de « mettre en place des mesures permettant la promotion des qualités innées et acquises de la population » (jinkō no sententeki, kötenteki shishitsu no kōjō 人口の先天的、 後天的資質の向上 ${ }^{40}$ ). Ainsi, en novembre 1946, fut élaboré le Nouvel Avis sur les mesures à tenir en matière de politique de population (Shinjinkō seisaku kihon hōshin ni kansuru kengi 新人口政策基本方針に関する建議) provenant du CMP. Le Nouvel Avis recommandait entre autres de légaliser la stérilisation involontaire, de prévoir des bourses pour les bons élèves, de répandre des services de conseil eugénique, et d'encourager les recherches en matière eugénique ${ }^{41}$.

37. Sugita, 2010, p. 207.

38. Sur la régulation de la sexualité du point de vue de la sauvegarde raciale durant cette période, voir KRAMm, 2017.

39. Matsubara, 1998a, p. 192-193.

40. Cité dans Sugita, 2010, p. 209.

41. Ibid., p. 201-202. 
Les débats politiques sur la qualité de la population affichaient des éléments de continuité et de rupture. Les acteurs étaient quasiment les mêmes que durant la période de guerre. Les figures centrales comprenaient : Nagai Tōru, dirigeant désormais le Comité sur les politiques de population au sein de la FRPP ; Koya Yoshio, présidant l'Institut national de la santé publique responsable devant le MSTAS ; Tachi Minoru 舘稔, démographe jouant un rôle clé aux côtés de Koya dans la rédaction du Plan; et enfin Nagai Hisomu qui, malgré son peu d'influence en raison de son âge avancé, continua à occuper des postes d'influence jusqu'à sa mort en 1957. Autrement dit, il y eut clairement une continuité chez les acteurs dirigeant les politiques de population d'avant jusqu'après la guerre.

En partie pour ces raisons, les débats politiques ainsi que les recommandations durant les premières années de l'après-guerre montrèrent des similitudes frappantes avec la période d'avant-guerre. Par exemple, le Nouvel Avis était pratiquement la copie des Diverses mesures concernant le contrôle de la population de 1929 mentionnées ci-dessus ${ }^{42}$.

Cela étant, les débats d'après-guerre différaient de ceux d'avant-guerre à plusieurs niveaux. Premièrement, nous assistons à des échanges croissants entre les militants en matière de contrôle des naissances et les conseillers en matière de population suite à l'entente entre le SCAP et le gouvernement japonais sur ce terrain ${ }^{43}$. Ainsi, Kitaoka Juitsu 北岡寿逸, l'une des figures centrales des mouvements pour le contrôle des naissances au Japon, fut nommé au sein du CCPP ; en outre, Katō Shizue 加藤シジエ, la doyenne desdits mouvements, devint l'une des premières femmes à siéger au sein de la Diète et contribua à la rédaction de la proposition du Parti socialiste de la loi de protection eugénique.

Deuxièmement, le changement générationnel permit à de nouvelles pensées de pénétrer dans les débats politiques. La période fut celle du déclin de l'influence de Nagai Hisomu qui s'approchait du terme de sa vie. En revanche, les chercheurs plutôt jeunes prirent de l'ampleur au sein des organismes «trinitaires » et apportèrent leur soutien à un concept alternatif rejoignant celui de Nagai Tōru d'avant-guerre. Nous pouvons ainsi citer le cas de Terao Takuma 寺尾讹

42. Ibid., p. 200-203.

43. Norgren, 2001 ; OAKley, 1978. 


\section{CIPANGO \\ 106 Cahiers d'études japonaises n² 24}

磨, économiste à l'université Keiō ${ }^{44}$. Dans les années 1930, Terao était déjà un chercheur confirmé dans les études de Malthus et avait publié des ouvrages fondateurs et influents dans les années 1940. Terao était alors conscient que les mesures eugénistes en vigueur ne permettaient pas un changement d'ampleur et nécessitaient des générations avant de produire un impact tangible sur la qualité de la population. Il soutint ainsi « un système éducatif et des modalités de vie rationnels » (gooriteki na kyōiku seido to seikatsu yōshiki 合理的な教育制度と生 活様式), qui devaient avoir un impact immédiat car s'adressant à l'ensemble de la population ${ }^{45}$.

Dans la période d'après-guerre, Terao fut désigné pour rejoindre le CMP. La théorie de Terao, similaire à l'euthénisme de Nagai Tōru, fut suivie par le CMP. Ainsi, en même temps que le changement générationnel chez les chercheurs, le sens de la qualité de la population se diversifia de nouveau dans la période d'après-guerre pour s'approcher de la compréhension large qui dominait dans les années 1920.

\section{Années 1960 : la qualité de la population au prisme du « développement social »}

La chute de la natalité à partir des années 1950 mena à une reconsidération de la qualité de la population ${ }^{46}$. Le gouvernement, y voyant l'aboutissement de sa politique de contrôle des naissances, s'en arrogea le mérite. Cependant, derrière ce succès, se cachaient des problèmes encore non résolus, à commencer par la mortalité des mères et enfants, encore élevée comparativement aux pays développés. En outre, malgré la campagne gouvernementale pour promouvoir le contrôle des naissances « moderne », il s'est avéré que le déclin du taux de

44. Outre Terao, Minoguchi Tokijirō offrit également un autre cas d'étude. Pour Minoguchi, voir Tamai \& Sugita, 2015.

45. Terao, 1941, p. 118-119.

46. En 1950, l'indice de fécondité au Japon était de 3,65, tandis qu'en 1960, il baissait à 2,0 . 
naissances était le fruit de l'avortement, lequel pouvait aggraver la mortalité des mères ainsi que la morbidité chez les enfants, déjà élevées ${ }^{47}$.

Le gouvernement et les experts finirent par considérer de concert qu'une réduction rapide de la fécondité compromettait la qualité de la population en déformant sa composition, et en générant par conséquent d'autres complications. La baisse de la population active était le point le plus sensible, d'autant plus qu'à la fin des années 1950, le Japon s'engageait dans une croissance économique sans précédent. Dans ce contexte, les experts du MSTAS agirent pour l'amélioration de la qualité de la population ${ }^{48}$.

Ainsi, à partir de 1958, des débats furent menés activement : la FRPP commença à lancer des recherches en accord avec les experts des ministères concernés ${ }^{49}$; en 1959, un groupe de recherche éclectique, dirigé par Nagai Tōru et comprenant Terao et Tachi, commença à mener des recherches grâce à des fonds accordés par le MSTAS. À cette même période, le CCPP du MSTAS commença à débattre sur la qualité de la population et chercha à rassembler les opinions des experts à ce sujet ${ }^{50}$. Un sous-comité au sein du CMP élabora les Mesures de base pour l'amélioration de la qualité de la population (Jinkō shishitsu kōjō ni kansuru taisaku yōkō 人口 資質向上に関する対策要綱) le 17 janvier 1962. Le CCPP rédigea quant à lui un projet à partir des recommandations de la FRPP et le publia en juillet 1962. Il en résulta qu'un chapitre entier dédié à la qualité de la population fut publié dans le Livre blanc de la population en $1963^{51}$.

L'individu fut alors déterminé comme l'élément constitutif de la population. Les Mesures du CMP définissaient en effet la qualité de la population comme l' « état de base de l'individu, qui, tant sur le plan mental que physique, détermine les caractéristiques de la population, composée d'un rassemblement d'hommes [...] susceptibles d'être modifiés artificiellement » (jin.iteki ni henkō kanō 人為 的に変更可能 $)^{52}$. Ainsi, à la différence de la période de guerre, ce processus ne

47. NORGREN, 1998, p. 59.

48. ZAIDAN HŌJIN JINKŌ MONDAI KENKYŪKaI, 1962, p. 13-14.

49. JINKŌ MONDAI SHINGIKAI, 1954.

50. WATANABE, 1960. Voir également Jinkō MONDAi SHingikaI, 9 août 1960.

51. Kösei hakusho, 1963 ; Gekkan shakai hoshō, 1963.

52. ZAIDAN HŌJIN JINKŌ MONDAI KENKYŪKAI, 1962, p. 29. 
108 Cahiers d'études japonaises n² 24

fut pas associé à la « race » dans les années 1960. Cela laissa la place à un espace discursif engageant davantage les efforts humains dans l'amélioration de la qualité de la population.

Par ailleurs, la compréhension de la qualité de la population ne se confondait pas avec celle d'avant-guerre en raison du discours sur le « développement » (kaihatsu 開発). La notion alors fréquemment empruntée pour qualifier le Japon était celle de 《 pays semi-développé » (chüshinkoku 中進国), un pays sujet à un développement social par le biais du « développement régional » (chiiki kaihatsu 地域開発) ou du “community development” (komyunitì deberoppumento コミュ ニティー・デベロップメント).

Notons toutefois que l'importance du développement des infrastructures est apparue en politique au milieu des années 1930, dans le contexte du Programme de développement et de promotion du Tōhoku (Tōhoku shinkō jigyō 東北振興 事業 $)^{53}$. À partir de la fin des années 1930, alors que les demandes en « ressources humaines » devinrent croissantes avec la guerre d'Asie-Pacifique, l'agenda politique prit au sérieux le « développement»des infrastructures régionales en santé publique et en bien-être pour enfants et femmes afin d'encourager les femmes à donner naissance à davantage d'enfants ${ }^{54}$.

L'idée que le développement puisse résoudre les problèmes de population revint dans le contexte de l'occupation. Le SCAP soutint un modèle de développement inspiré du projet de la Tennessee Valley Authority (TVA) qui avait été donné en réponse aux problèmes liés à la surpopulation (chômage, niveau de vie, etc.). La vision du SCAP fut reçue favorablement par l'administration japonaise qui cherchait à obtenir une indépendance nationale à travers la reconstruction économique. Par conséquent, le gouvernement japonais promut le sōgō kaihatsu 総合開発, soit le 《développement global », à l'origine d'une série de directives étatiques à portée large qui, prenant modèle sur la TVA, encouragea par exemple la construction des barrages ${ }^{55}$.

53. L'un des premiers programmes étatiques de développement économique et d'infrastructures dans une région en particulier, il fut mis en place en 1936 suite à la famine qui frappa la région du Tōhoku en 1934. Voir Dinmore, 2013, p. 10-12.

54. Kawauchi, 2015, p. 7. Pour une vue plus générale : Fujino, 2003.

55. Dinmore, 2013. 
Toutefois, au cours des années 1950, le développement, jusque-là principalement prôné en matière économique, fut l'objet de critiques croissantes. Cela poussa les Nations unies à créer un nouveau concept complémentaire, celui de « développement social », « fondamental pour atteindre la paix et la sécurité internationales et une croissance bénéfique rapide et mutuelle de la prospérité du monde ». En décembre 1961, l'Assemblée générale désigna les années 1960 comme la première décennie de l'ONU, et elle fut consacrée au développement, les États membres étant encouragés à « intensifier leurs efforts (...) vers un avancement social ${ }^{56} \gg$.

Dans ce contexte, l'expert Tachi Minoru, avec Ibe Hideo 伊部英男, spécialiste en politique sociale, traduit le terme "social development" en japonais par shakai kaihatsu 社会開発, défini à son tour comme le « développement des aspects sociétaux des zones urbaines et rurales, habitat, transport, santé, médecine, hygiène publique et environnementale, bien-être social, éducation, etc. ». Le développement social fut soigneusement juxtaposé au développement économique, défini quant à lui comme le « développement de diverses industries centrées sur leur dimension économique ${ }^{57} \gg$.

La nouvelle dimension sociale reconnue au développement répondait aux inquiétudes ressenties au sein du MSTAS face à la prédominance économique du programme du « développement général ». En effet, ce mode de développement, favorable aux travaux d'infrastructures et à l'industrie lourde, était susceptible de causer à terme de graves problèmes environnementaux et sociaux tels que la pollution. Dans ces circonstances, le MSTAS commença à mettre l'accent sur le besoin d'intégrer le développement social dans ses mesures de santé et de bienêtre $^{58}$. Dans son discours inaugural de novembre 1964, le nouveau Premier ministre Satō Eisaku 佐藤栄作 expliquait le développement social comme des « efforts pour promouvoir et développer un niveau de vie décent dans une société contemporaine qui, sujette à un développement gigantesque de l'économie et de la technologie, tend à perdre de vue l'existence de l'homme ». Partant de ce constat,

56. The United Nations General Assembly, 1961.

57. JinKō MONDAI SHINGIKaI, 1964, p. 165-167. Pour une description plus détaillée de la façon dont l'expression fut introduite au Japon, voir SUGITA, 2015b, p. 249.

58. Matsubara, 1998b, p. 192. 


\section{CIPANGO}

110 Cahiers d'études japonaises $n^{\circ} 24$

Satō fit du développement social la base de sa politique ${ }^{59}$. Après son discours, le 11 décembre 1964, Takahashi Mamoru 高橋衛, secrétaire à la planification économique, soumit à Satō le Plan basique du développement social (Shakai kaihatsu ni tsuite no kihon kōsō 社会開発についての基本構想). Ce plan soulignait l'intégration du développement social dans le développement régional (chiiki kaihatsu 地域開発) ${ }^{60}$. Se référant à ce Plan, le budget gouvernemental de 1965 fut alloué prioritairement aux mesures promouvant le développement social lié au redressement des structures économiques, à la diminution des écarts entre les régions et au problème de surpeuplement des centres urbains ${ }^{61}$.

C'est dans ce contexte que le développement fut relié à la qualité de la population : au sein du CMP de la FRPP, la qualité de la population fut définie comme le marqueur indicatif du succès japonais en développement. Fondé sur cette compréhension, les Mesures du CMP (1962) félicitèrent la rapidité par laquelle le développement du pays eut lieu en l'espace d'une décennie après la guerre tout en insistant sur les conséquences néfastes d'un développement précipité sur la qualité de la population. En effet, les Mesures rappelaient que, malgré la rapidité de la croissance économique, encore $40 \%$ de la main-d'œuvre totale étaient consacrés à l'industrie primaire, à la différence des pays développés où l'industrie était déjà passée aux industries secondaire et tertiaire. En outre, la croissance économique était maintenue non par les «grandes entreprises modernes » (kindaiteki daikigyō 近代的大企業) mais par les 《 petites et moyennes entreprises non modernes 》 (bikindaiteki chūshō kigyō 非近代的中小企業) qui dépendaient d'une « jeune main-d'œuvre importante, non qualifiée et mal payée ».

Les Mesures soulignaient par ailleurs que l'évolution démographique japonaise pouvait ressembler à celle des pays développés, en particulier en raison de sa transition démographique récente et réussie avec une baisse drastique de la fécondité. Toutefois, la transition japonaise avait été accomplie grâce àl'avortement, de façon non planifiée, à la différence des pays développés où la transition fut le fruit du «planning familial moderne » (kindaiteki kazoku keikaku 近代的家族 計画) qui impliquait une planification rationnelle de la taille familiale à l'aide

59. Asahi shinbun, 21 novembre 1964.

60. Asahi shinbun, 12 décembre 1964.

61. Asahi shinbun, 18 décembre 1964. 
des méthodes contraceptives. Sur cette base, les Mesures concluaient que le Japon avait certes fait une transition réussie vers le statut d'État « semi-développé », mais que celle-ci fut menée à la hâte, sans réforme socioéconomique fondamentale comme ce fut le cas dans les pays développés. Ainsi, poursuivaient-elles, le pays avait encore besoin de mesures correctives spéciales avant de devenir un pays réellement développé ${ }^{62}$.

Se fondant sur les débats susvisés au sein de la FRPP, les membres du CCPP réaffirmèrent l'importance du développement social dans les régions rurales et provinciales dans la perspective d'une amélioration de la qualité de la population. Ils se focalisèrent alors sur le déclin de la population en tant que facteur compromettant la qualité de la population. Dans son mémorandum soumis au MSTSA le 18 août 1963, la FCPP avertit que le développement économique dans les années 1950 avait provoqué un grand rassemblement migratoire à un niveau international vers les pôles industriels, venant en conséquence appauvrir la population des jeunes hommes déracinés de leur milieu rural, lequel était sujet à son tour à un vieillissement et une féminisation de sa population. Le mémorandum expliquait ensuite que le déclin de la population n'était pas un problème propre aux zones rurales, étant précisément l'écart entre la population urbaine et les communautés rurales à l'origine de la « destruction de la capacité reproductive » de la population japonaise (saiseisan nōryoku wo kōhai saseru 再生産能力を荒 廃させる) $)^{63}$.

Se fondant sur cette compréhension, selon le mémorandum, le gouvernement devait s'efforcer d'atteindre un équilibre entre le développement économique et social afin de conserver une haute qualité de sa population, et le développement régional était un moyen efficace d'atteindre cet objectif. Il recommandait ainsi que le gouvernement prenne en compte entre autres le vieillissement, l'aménagement des villes, la pollution, les relations locales, ou le développement régional.

Cela déclencha des initiatives dans le développement social. En novembre 1964, le gouvernement Satō mit en place un Cercle de discussion sur le développement social (Shakai kaihatsu kondankai 社会開発懇談会) au sein du Cabinet, ainsi qu'une entité indépendante, le Bureau de la vie du peuple (Kokumin seikatsukyoku

62. ZAIDAN HŌJIN JINKŌ MONDAI KENKYŪKAI, 1962, p. 7-10.

63. JINKŌ MONDAI SHINGIKAI, 1964, p. 2. 
112 Cahiers d'études japonaises nº 24

国民生活局), au sein du Cabinet ministériel. Dans son Livre blanc de 1964, le Bureau énonçait que le développement social était un sujet important auquel le gouvernement devrait s'attaquer dans le futur.

Le discours de développement au début des années 1960 permet de faire ressortir deux remarques sur la compréhension de la politique de la population au Japon. La première remarque concerne sa continuité historique. D'un côté, en se concentrant sur le développement, on identifie un modèle de gouvernance propre à l'après-guerre, marqué par un nouveau mode de pensée. D'un autre côté, cela se fit sur fond d'anciennes idéologies existantes, la promotion des « mariages sains » (kenzen na kekkon 健全な結婚) ${ }^{64}$ ou encore la prévention des mauvais gènes en étant des exemples éloquents. Cependant, ces recommandations eugénistes avaient une moindre importance comparativement aux mesures plus fondamentales touchant les politiques sociales et de bien-être. Il conviendrait alors de considérer le mode de gouvernance de la population dans le Japon moderne sous l'angle de l'accumulation plutôt qu'en termes de rupture et continuité : le processus politique dans la période d'après-guerre était fondé sur les idéologies préexistantes telles que l'eugénisme et l'euthénisme, mais il absorba en même temps les discours relatifs au développement qui vint s'ajouter comme une couche additionnelle.

Une deuxième remarque vient expliciter la connexion entre la politique interne et la politique externe du Japon. En 1952, le Japon devint de nouveau une nation indépendante après le Traité de paix de San Francisco. En outre, en 1956, il revint sur la scène internationale en rejoignant les Nations unies. Cependant, dans les années 1960, on pouvait difficilement soutenir que le Japon était un leader politique mondial. Économiquement, certes le pays traversait une haute croissance économique. Toutefois, ce ne fut pas avant 1975 qu'il devint membre des nations industrialisées ${ }^{65}$. Dans ce contexte, l'une des missions les plus importantes du Japon fut d'asseoir sa position internationale par le biais des aides internationales pour le développement, alors que la modernisation via le développement socioéconomique était devenue un outil diplomatique majeur sous la Guerre froide ${ }^{66}$.

64. Asahi shinbun, 25 juin 1965.

65. Satō \& Shimomura, 2012.

66. LАTHAм, 2011. 
C'est dans ce contexte historique que l'appel du gouvernement Satō pour le développement social fut lancé, en coïncidence avec les efforts gouvernementaux encore tâtonnants dans le développement des aides internationales à travers la coopération technique et médicale. En effet, le gouvernement commença alors à s'engager dans le planning familial dans le cadre des aides au développement (ODA), dont le destinataire premier fut l'Asie ${ }^{67}$.

Ces deux phénomènes - la montée de l'appel pour l'amélioration de la qualité de la population au Japon et la participation au planning familial sur la scène internationale - peuvent paraître sans lien. Or, nous considérons qu'il ne s'agit pas d'une pure coïncidence, précisément parce que ces deux événements partagent le même point de départ, à savoir le besoin d'asseoir le statut du Japon parmi les pays développés, non seulement en tant que puissance économique, mais aussi via son développement social. Il est à noter que l'image d'un Japon « semidéveloppé » fut alors exploitée dans le domaine du planning familial pour insister sur l'unicité de l'aide japonaise : le Japon serait un donneur particulièrement efficace étant proche des pays receveurs, comparé aux autres donneurs dont la majorité est constituée de pays occidentaux « développés » ayant accompli leur développement socioéconomique depuis longtemps. Ainsi, même ce statut de « semi-développé » permit au Japon d'ancrer ses efforts dans le domaine diplomatique.

Ces deux stratégies, internes et externes, s'entrecroisèrent autour de l'identification des régions nécessitant le développement. Dans le cas de la campagne japonaise pour la dénatalité dans les pays en voie de développement, le gouvernement japonais se focalisa avant tout sur l'Asie. Cette décision se fondait sur la théorie de la transition démographique qui fournissait une justification théorique au mouvement transnational de contrôle de la population pour considérer l'Asie comme «la $»$ région nécessitant des initiatives en matière de planning familial ${ }^{68}$. Les partisans des aides extérieures au développement adoptèrent cette perspective pour faire de l'Asie une région sous-développée, et

67. Номеi, 2016.

68. Pour le dire simplement, la théorie de la transition démographique soutenait que les sociétés avec une population importante fonctionnant à partir d'une natalité haute et une mortalité haute étaient à la première étape de leur transition démographique. 


\section{CIPANGO}

114 Cahiers d'études japonaises nº 24

décidèrent de se concentrer sur cette région ${ }^{69}$. En même temps, dans les politiques nationales visant à promouvoir la qualité de la population par le « développement régional », la région périphérique du Tōhoku, ainsi que, dans une certaine mesure, le sud de Kyūshū furent identifiés comme ayant un besoin particulier en termes de développement. Les études démographiques soutinrent cette vision, parlant du Tōhoku comme étant le « Tibet du Japon ${ }^{70} »$. Cette perspective, qui compare le Tōhoku au Tibet, alors internationalement identifié comme l' « arrière-pays » de l'Asie, fut directement formée par le discours mondial du développement. Celui-ci, fusionné avec la théorie de modernisation, afficha la vision du monde qui devait quitter son passé arriéré pour entrer dans un futur moderne et brillant. Ainsi, dans ce sens également, les efforts du Japon sur les plans national et international, a priori sans connexion, étaient en réalité extrêmement liés, les deux cherchant à contribuer à une plus grande reconnaissance mondiale du Japon.

\section{Conclusion}

L'intérêt politique pour la qualité de la population fut éphémère. Au cours des années 1970, étant donné que la qualité de la population s'articulait avec la politique étrangère, elle fut de plus en plus intégrée dans d'autres problématiques telles que les politiques sanitaire, sociale, environnementale et de bien-être, ce qui rendit difficile d'établir une politique cohérente à cet égard. À la fin des années 1970, seuls les experts en matière de population employaient l'expression « qualité de population », et, même dans le domaine des sciences de la population, les chercheurs commencèrent à déplacer leur centre d'intérêt ailleurs. Vers le milieu des années 1980, la qualité de la population commença à tomber dans l'oubli.

Le présent travail examine ainsi le développement du concept de qualité de la population entre les années 1910 et les années 1960. Des termes autres que l'eugénisme avaient dominé la scène politique avec pour objectif d'améliorer la qualité de la population. En effet, d'un côté, fut promue une pensée eugéniste

69. Un autre facteur très important, qui ne sera pas approfondi dans le présent article faute de temps, est le problème de la réparation des dommages causés par la guerre. Voir Asano, 2013.

70. UсHino, 1966, p. 82. 
fondée sur des considérations raciales par Nagai Hisomu et Koya Yoshio, la qualité de la population dans ce contexte étant synonyme de qualité de la race japonaise. L'individu en tant qu'être biologique en était le point de départ, sa capacité reproductive le facteur déterminant. Les propositions politiques étaient alors en lien direct avec les politiques de santé, et elles furent étroitement liées au MSTAS.

D’un autre côté, une notion plus large de qualité de la population, avancée par Nagai Tōru et des scientifiques sociaux tels que Terao Takuma, fut présente dans les débats politiques à partir des années 1920. Cette version impliquait l'euthénisme, soit la promotion des conditions entourant la vie en général. Les propositions issues de ce concept large étaient plus englobantes, s'intéressant non seulement à la santé mais aussi aux politiques sociales et de bien-être en lien avec les activités humaines. Cette conception large se développa en conjonction avec l'idéologie eugéniste.

En retraçant ce développement historique, le présent travail cherche à identifier les éléments de continuité et de rupture avant et après 1945 afin de présenter une vision qui se veut globale des efforts du gouvernement japonais en matière de population. Il montre d'une part que la configuration entre l'eugénisme et l'euthénisme fut déterminante pour définir le cadre des débats gouvernementaux sur la qualité de la population depuis les années 1920. Cette configuration pouvait dépendre des individus qui exerçaient le pouvoir dans le processus politique. Les conseillers politiques tels que Nagai Tōru montèrent en pouvoir dans les années 1920 et continuèrent à exercer une influence pendant la période d'après-guerre. La continuité des personnes est un facteur indéniable pour connecter les pensées politiques avant et après 1945, et elle permet d'approfondir la compréhension de la construction des débats et des propositions durant cette période.

D'un autre côté, nous tentons de montrer combien le concept de développement, redéfini et en plein essor, complexifia la gouvernance de la population d'après-guerre. Il est particulièrement intéressant de voir que les partisans du développement exploitèrent à cette fin l'image auto-attribuée de pays « semi-développé », ainsi que celle d' « arrière-pays » sur les plans interne et externe, soit respectivement la région du Tōhoku et l'Asie. Le développement n'indique pas seulement le point de départ des débats d'après-guerre sur la 


\section{CIPANGO}

116 Cahiers d'études japonaises n² 24

population mais il nous incite également à analyser la façon dont les débats sur la population nationale furent influencés par les politiques internationales.

Le présent article n'est pas une histoire intellectuelle des acteurs élites de la société même si nous ne pouvons pas nier l'appartenance des protagonistes à des classes et responsabilités élevées. À travers l'histoire moderne du Japon, nous pouvons surtout voir que les décisions, prises par cette petite poignée d'élites, avaient des conséquences considérables sur la vie de tous les jours du peuple japonais en général. L'histoire n'a pas de fin. Sous l'expression « dénatalité et vieillissement » (shōshi kōreika), les propositions politiques fusent, les mesures prises affectent quasiment tout le pays. Dans ce contexte, l'analyse de l'histoire de la qualité de la population semble particulièrement pertinente car elle nous incite à réfléchir sur la façon dont l'État considère et tente de diriger la vie des citoyens japonais au nom de la population et de sa qualité.

\section{Bibliographie}

Asahi shinbun 朝日新聞, 21 novembre 1964, « Satō shushō, hatsu no shoshin hyōmei » 佐藤首相、初の所信表明 (Première déclaration d'intention du Premier ministre Satō), édition du soir.

Asahi shinbun 朝日新聞, 12 décembre 1964, « Mazu jūku nado nanatsuno kōmoku. Shushō ryōshō. Shakai kaihatsu no kihon kōsō»まず住区な ご七つの項目 首相了承 社会開発の基本構想 (Cadre de base du développement social, sept éléments dont l'habitat, acceptés par le Premier Ministre), édition du jour.

Asahi Shinbun 朝日新聞, 18 décembre 1964, «Sekkyokuteki ni shakai kaihatsu 》 積極的に社会開発 (Développement social actif), édition du soir.

Asahi shinbun 朝日新聞, 25 juin 1965, « Gakureki no henchō wo yameyo, jūtaku seisaku wo nerinaose. Shakai kaihatsu kondankai, chūkan hōkoku no yōshi 》学歴の偏重をやめよ、住宅政策を練り直せ 社会開発懇談 会中間報告の要旨 (Mettons fin aux déséquilibres scolaires, reformulons 
les politiques de l'habitat, Cercle de discussion sur le développement social, résumé du rapport intermédiaire), édition du jour.

Asano Toyomi 浅野豊美 (dir.), 2013, Sengo Nihon no baishō mondai to higashi Ajia no chiiki saiben 戦後日本の賠償問題と東アジアの地域再編 (Le problème japonais d'indemnisation d'après-guerre et la restructuration de la région de l'Asie de l'Est), Jigakusha shuppan 慈学社出版, Tōkyō.

Bashford Alison \& Levine Philippa (ed.), 2010, The Oxford Handbook of the History of Eugenics, Oxford University Press, Oxford.

Chūman Mitsuko 中馬充子, 2013, « Nagai Hisomu saikō : yūseigaku keimō katsudō no shinsō wo saguru »永井潜再考-優生学啓蒙活動の真相 を探る (Nouvelle réflexion sur Nagai Hisomu : la véritable nature de ses activités de vulgarisation de l'eugénisme), in YAMAZAKI Kiyoko 山崎喜代子 (dir.), Seimei no rinri : Yūsei seisaku no keifu 生命の倫理 優生政策の系譜 (L'éthique de la vie : la généalogie des politiques eugénistes), Kyūshū daigaku shuppankai 九州大学出版会, Fukuoka, p. 225-267.

Chung Yuehtsen Juliette, 2002, Struggle for National Survival: Eugenics in SinoJapanese Contexts, 1896-1945, N.Y. Routledge, New York.

Chung Yuehtsen Juliette 鐘月岑, 2013, « Nihon no senryōchi seisakuka ni okeru yūseigaku »日本の占領地政策化における優生学 (L'eugénisme sous l'occupation japonaise), in Yamazaki Kiyoko (dir.), Seimei no rinri: Yüsei seisaku no keifu (L'éthique de la vie : la généalogie des politiques eugénistes), Kyūshū daigaku shuppankai, Fukuoka, p. 269-316.

Connelly Matthew James, 2008, Fatal Misconception: The Struggle to Control World Population, Belknap Press of Harvard University Press, Cambridge, Mass., London. 


\section{CIPANGO}

118 Cahiers d'études japonaises n² 24

Dinmore Eric, 2006, A Small Island Nation Poor in Resources: Natural and Human Resource Anxieties in Trans-World War II Japan, Ph.D. dissertation, Princeton University.

Dinmore Eric, 2013, "Concrete Results?: The TVA and the Appeal of Large Dams in Occupation-Era Japan," The Journal of Japanese Studies, vol. 39, $\mathrm{n}^{\circ} 1$, January 31, p. 1-38.

Donaldson Peter J., 1990, Nature against Us: The United States and the World Population Crisis, 1965-1980, University of North Carolina Press, Chapel Hill.

Escobar Arturo, 1995, Encountering Development: The Making and Unmaking of the Third World, New edition, Princeton Studies in Culture/Power/History, N.J Princeton University Press, Princeton.

FrÜHSTÜCK Sabine, 2003, Colonizing Sex: Sexology and Social Control in Modern Japan, University of California Press, Berkeley.

Fuchs Stephen J., 2007, "Feeding the Japanese: Food Policy, Land Reform, and Japan's Economic Recovery," in Mark E. Caprio \& Sugita Yoneyuki (ed.), Democracy in Occupied Japan: The U.S. Occupation and Japanese Politics and Society, Routledge, London, p. 26-47.

FujIme Yuki 藤目ゆき, 1997, Sei no rekishigaku : kōshō seido, dataizai taisei kara baishun bōshihōo, Yūsei hogohō taisei e 性の歴史 公娼制度・值胎罪 体制から売春防止法・優生保護法体制へ(L'histoire de la sexualité, du système prostitutionnel et de la pénalisation de l'avortement à la loi relative à la protection eugénique), Fuji shuppan 不二出版, Tōkyō.

Fujino Yutaka 藤野豊, 1998, Nihon fashizumu to yüsei shisō 日本ファシズ ムと優生思想 (La pensée eugéniste et le fascisme japonais), Kamogawa shuppanかもがわ出版, Kyōto. 
Fujıno Yutaka, 2003, Kōseishō no tanjō : Iryō wa fashizumu wo ikani suishin shitaka 厚生省の誕生一医療はファシズムをいかに推進したか, Kamogawa shuppan, Kyōto.

Gekkan shakai hoshō 月刊社会保障, 1963, 《Jinkō shishitsu no kōjō wo teiki »人口資質の向上を提起 (Proposer la promotion de la qualité de la population), no 193 , janvier, p. 82-87.

Hiroshima Kiyoshi 廣嶋清志, 1980, « Gendai Nihon jinkō seisakushi shōron » 現代日本人口政策史小論 (Histoire contemporaine des politiques de population japonaises), Jinkō mondai kenkyū 人口問題研究, $n^{\circ} 154$, avril, p. 46-61.

Hiroshima Kiyoshi 広島清志, 1981, « Gendai Nihon jinkō seisakushi shōron 2 » 現代日本人口政策史小論2 (Histoire contemporaine des politiques de population japonaises 2), Jinkō mondai kenkyū 人口問題研究, $n^{\circ} 160$, octobre, p. 61-77

Homer Aya, 2016, "Between the West and Asia: 'Humanistic' Japanese Family Planning in the Cold War," East Asian Science, Technology and Society, vol. 10, n 4, December 1, p. 445-467.

Hopson Nathan, 2017, Ennobling Japan's Savage Northeast: Tōhoku as Japanese Postwar Thought, 1945-2011, Harvard University Asia Center, Harvard, Massachusetts.

JINKŌ MONDAI SHINGIKAI 人口問題審議会 (Conseil consultatif sur les problèmes de population), 1954, Daisankai Jinkō mondai shingikai giji sokkiroku 第三回人口問題審議会議事速記録 (Minutes de la $3^{\mathrm{e}}$ réunion du Conseil consultatif sur les problèmes de population), 22 janvier. (URL: http://www.ipss.go.jp/history/shingikai/data/PDF2009120131.pdf)

Jinkō MONDAI SHINGIKAI, 1960, Jinkō mondai shingikai dainijūikkai sōkai sokkiroku人口問題審議会第二十一回総会議事速記録 (Minutes de la 


\section{CIPANGO}

120 Cahiers d'études japonaises nº 24

$21^{\mathrm{e}}$ réunion générale du Conseil consultative sur les problèmes de population), 9 août.

JINKŌ MONDAI SHINGIKAI, 1962, Jinkō shishitsu kōjō taisaku ni kansuru ketsugi 人口資質向上対策に関する決議 (Résolution relative aux mesures pour la promotion de la qualité de la population), Kōseishō, 12 juillet.

Jinkō MONDAI SHINGIKaI, 1964, « Chiiki kaihatsu ni kanshi, jinkō mondai no kenchi kara tokuni ryūisubeki jikō ni tsuiteno iken » 地域開発に関 し冈人口問題の見地から特に留意すべき事項についての意見 (Avis sur les points problématiques concernant la population du point de vue du développement régional), in KōsEISHO DAIJIN KANBŌ KIKAKUSHITSU 厚 生省大臣官房企画室 (dir.), Jümin no seikatsu to shinsangyō toshi 住民の 生活と新産業都市 (La vie des habitants et les nouvelles villes industrielles), Ōkurashō insatsushitsu 大蔵省印刷室, Tōkyō, p. 165-167. (URL: http:// www.ipss.go.jp/history/shingikai/data/J000008916.pdf).

JINKŌ SHOKURYŌ MONDAI CHŌSAKAI 人口食糧問題調査会 (Commission d'enquête sur la population et le ravitaillement), 1930, Jinkō shokuryō mondai chōsakai jinkōbu tōshin setsumei 人口食糧問題調查会人口部答申説明 (Commission d'enquête sur la population et le ravitaillement, section de la population, commentaires), Jinkō shokuryō chōsakai, Tōkyō.

KaWAUchi Atsushi 川内淳史, 2015, « Jinkō to Tōhoku-Senjiki kara sengo ni okeru Tōhoku “kaihatsu” tono Kanren de »人口と東北一戦時期から 戦後における東北「開発」との関連で一 (Population et Tōhoku en relation avec le « développement » du Tōhoku durant et l'après-guerre), in Yамамото Takenori 松本武祝 (dir.), Tōhoku chibō "kaihatsu" no keifu - Kindai no sangyō seisaku kara Higashi Nibon daishinsai made 東北 地方「開発」の系譜一近代の産業政策から東日本大震災まで一 (La généalogie du « développement »du Tōhoku - depuis les politiques industrielles modernes jusqu'au séisme du 11 mars 2011), Akashi shoten 明石 書店, Tōkyō, p. 1-17. 
Kōsei hakusho厚生白書 (Livre blanc de la Santé), 1963, Kōseishō 厚生省, Tōkyō. (URL: http://www.mhlw.go.jp/toukei_hakusho/hakusho/kousei/1962/)

Koya Yoshio 古屋芳雄, 1941, Kokudo, jinkō, ketsueki 国土・人口・血液 (Territoire, population, sang), Asahi shinbunsha 朝日新聞社, Tōkyō, p. 40.

Kramm Robert, 2017, Sanitizing Sex: Regulating Prostitution, Venereal Disease, and Intimacy in Occupied Japan, 1945-1952, University of California Press, Berkeley.

Kuвo Hidebumi 久保秀史, 1997, Nihon no kazoku keikakushi : Meiji, Taishō, Shōwa 日本の家族計画史 明治 - 大正 - 昭和 (Le planning familial au Japon : Meiji, Taishō, Shōwa), Shadan hōjin Nihon kazoku keikaku kyōkai 社 団法人日本家族計画協会, Tōkyō.

Latham, Michael E., 2011, The Right Kind of Revolution: Modernization, Development, and U.S. Foreign Policy from the Cold War to the Present, Cornell University Press, Ithaca.

Matsubara Yōko, 1998a, “The Enactment of Japan's Sterilization Laws in the 1940s: A Prelude to Postwar Eugenic Policy”, Historia Scientiarum 8, n 2 , p. 187-201.

Matsubara Yōko 松原洋子, 1998b, Nihon ni okeru yūsei seisaku no keisei 日 本における優生政策の形成 (La formation des politiques eugénistes au Japon), thèse, université d'Ochanomizu お茶の水大学.

Matsubara Yōko, 2000, « Nihon-sengo no yūsei hogohō to iu nano danshuhō »日本戦後の優生保護法という名の断種法 (Loi relative à la protection eugénique, une loi de stérilisation dans le Japon d'après-guerre), in Yonemoтo Shōhei 米本昌平 et al., Yūseigaku to ningen shakai 優生学 と人間社会 (Eugénisme et sociétés humaines), Kōdansha 講談社, Tōkyō, p. $169-236$. 


\section{CIPANGO}

122 Cahiers d'études japonaises nº 24

Matsubara Yōko, 2002, "Introduction to History of Science: Historical Analysis of the Engenic Laws in Japan”, Kagakushi Kenkyū 科学史研究, vol. 41, n² 222, June, p. 104-106.

McCAnn Carole R., 2016, Figuring the Population Bomb: Gender and Demography in the Mid-Twentieth Century, University of Washington Press, Seattle.

Norgren Tiana, 1998, "Abortion Before Birth Control: The Interest Group Politics Behind Postwar Japanese Reproduction Policy", The Journal of Japanese Studies, 24, no 1, p. 59-94.

Norgren Tiana, 2001, Abortion before Birth Control: The Politics of Reproduction in Postwar Japan, Princeton University Press, Princeton.

OAKLEY Deborah, 1978, "American-Japanese Interaction in the Development of Population Policy in Japan, 1945-52", Population and Development Review 4, $n^{\circ} 4$, p. 617-643.

ŌBAYASHi Michiko 大林道子, 2006, « Sengo Nihon no kazoku keikaku fukyū katei ni kansuru kenkyū » 戦後日本の家族計画普及過程に関する研究 (Recherches relatives au processus de diffusion du planning familial d'aprèsguerre au Japon), thèse, université d'Ochanomizu, Tōkyō.

O'Bryan Scott, 2009, The Growth Idea: Purpose and Prosperity in Postwar Japan, University of Hawaii Press, Honolulu.

OGINo Miho 荻野美穂, 2008a, «Kazoku keikaku » eno michi 家族計画への 道 (La voie vers le «planning familial »), Iwanami Shoten 岩波書店, Tōkyō.

Ogino Miho, 2008b, « Jinkō seisaku no sutoratejīi, "Umeyo fuyaseyo" kara “kazoku keikaku e” 》人口政策のストラテジー「産めよ殖やせよ」か ら「家族計画」へ(La stratégie des politiques de population, des mesures natalistes au planning familial), in TACHI Kaoru 舘かおる (dir.), Tekuno/ baio poritikkusu - Kagaku iryō gijutsu no ima テクノ/バイオ・ポリテ 
イクスー科学医療技術の今一 (Techno/biopolitique - les techniques scientifiques et médicales d'aujourd'hui), Sakuhinsha 作品社, Tōkyō, p. 145-159.

OGUMA Eiji 小熊英二, 1994, « Tsumazuita junketsu shugi - yūseigaku seiryoku no minzoku seisakuron » 躓いた純血主義一優生学勢力の民族政策 論 (La théorie du sang pur à l'épreuve - les politiques raciales menées par les pouvoirs eugénistes), Jökyō 情況, $\mathrm{n}^{\circ} 11$, p. 38-50.

Oguma Eiji, 1995, Tan.itsu minzoku shinwa no kigen 単一民族神話の起源 (Aux origines du mythe du peuple homogène), Shin.yōsha 新曜社, Tōkyō.

Отsuвo Sumiko, 1999, "Feminist Maternal Eugenics in Wartime Japan," U.S.Japan Women's Journal. English Supplement, n 17, p. 39-76.

Robertson Jennifer, 2001, “Japan's First Cyborg? Miss Nippon, Eugenics and Wartime Technologies of Beauty, Body and Blood”, Body \& Society, vol. 7, $\mathrm{n}^{\circ}$ 1, mars, p. 1-34.

Robertson Jennifer, 2010, "Eugenics in Japan: Sanguinous Repair," in Alison Bashrord \& Philippa Levine (ed.), The Oxford Handbook of the History of Eugenics, Oxford University Press, Oxford, p. 430-448.

Robertson Jennifer, 2012, "Hemato-Nationalism: The Past, Present, and Future of 'Japanese Blood'”, Medical Anthropology, vol. 31, nº 2, p. 93-112.

SATŌ Jin 佐藤仁, 2011, «Motazaru kuni » no shigenron : jizoku kanō na kokudo wo meguru moubitotsu no chi「持たざる国」の資源論一持続可能な国 土をめぐるもう一つの知一 (À propos des ressources d'un « pays qui n'en a pas » - une autre sagesse autour d'un territoire durable), Tōkyō daigaku shuppankai 東京大学出版会, Tōkyō. 


\section{CIPANGO}

124 Cahiers d'études japonaises n² 24

Satō Jin \& Shimomura Yasutami (eds), 2012, The Rise of Asian Donors: Japan's Impact on the Evolution of Emerging Donors, Routledge-GRIPS Development Forum Studies 1, N.Y. Routledge, Abingdon, Oxon, New York.

SCHAFfner Karen J. (ed.), 2014, Eugenics in Japan, Kyūshū daigaku shuppankai, Fukuoka.

SHINOZAKI Nobuo 篠崎信男, 1979, « Jinkō mondai no shitsuteki henbō » 人口問題の質的変貌 (La transformation qualitative des problèmes de population), Jinkō mondai kenky ū 人口問題研究, $\mathrm{n}^{\circ} 152$, octobre, p. 17-26.

Sugita Naho 杉田菜穂, 2010, Jinkō, kazoku, seimei to shakai seisaku - Nihon no keiken - 人口・家族・生命と社会政策一日本の経験一 (Population, famille, vie et politiques sociales - l'expérience japonaise), Hōritsu bunkasha, Kyōto.

Sugita Naho, 2013, <Yūsei >, <yūkyō > to shakai seisakujinkō mondai no Nihonteki tenkai <優生 $>$ ・ <優境 $>$ と社会政策一人口問題の日本的展開一 (<Eugénisme> <euthénisme> et politiques sociales - le développement à la japonaise des problèmes de population), Hōritsu bunkasha 法律文化社, Kyōto.

Sugita Naho, 2015a, « Nihon ni okeru jinkō shishitsu gainen no tenkai to shakai seisaku : senzen kara sengo e »日本における人口資質概念の展開と社 会政策一戦前から戦後へ- (Le développement du concept de qualité de la population et les politiques sociales au Japon - d'avant-guerre à aprèsguerre), Keizaigaku zasshi 経済学雑誌 vol. 116, n² 2, septembre, p. 59-81.

Sugita Naho, 2015b, « Nihon ni okeru shakai kaihatsuron no keisei to tenkai »日 本における社会開発論の形成と展開 (La formation et le développement du discours sur le développement social au Japon), Jinkō mondai kenkyū 人口 問題研究, vol. 71, nº 3, septembre, p. 241-259. 
Suzuki Zenji 鈴木善次, 1983, Nihon no yüseigaku 日本の優生学 (L'eugénisme au Japon), Sankyō shuppan 三共出版, Tōkyō.

TAmA Yasuko田間泰子, 2001, Boseiai to iu seido - Kogoroshi to chüzetsu no poritikkusu 母性愛という制度一子殺しと中絶のポリティクスー (Institution dite amour maternel - Politiques autour de l'infanticide et de l'avortement), Keisō shobō 勁草書房, Tōkyō.

TAmA Yasuko, 2006, « Kindai kazoku 》 to bodī poritikkusu 近代家族とボデ イ・ポリティックス (La 《 famille moderne » et la biopolitique), Sekai shisōsha 世界思想社, Kyōto.

TAma Yasuko, 2014, « “Umu, umenai, umanai” to Nihon no sengo »「産む・ 産めない・産まない」と日本の戦後 (《Accoucher, ne pas pouvoir accoucher, ne pas accoucher », l'après-guerre au Japon), in KoHAmA Masako 小浜正子, MATsuoka Eiko 松岡悦子 (dir), Ajia no shussan to kazoku keikakuアジアの出産と家族計画 (L'accouchement en Asie et le planning familial), Bensei shuppan 勉成出版, Tōkyō, p. 27-62.

TAMA Yasuko, 2016, « Jutai chōsetsu (bāsu kontorōru) to botai hogohō » 受胎調 節（バース・コントロール） と母体保護法 (Le contrôle des naissances et la loi relative à la protection du corps maternel), in SHIRAI Chiaki 白井千 晶 (dir.), Umi sodate to josan no rekishi - Kindaika no 200-nen wo furikaeru 産 み育てと助産の歴史一近代化の 200 年をふり返るー (L'histoire de la reproduction et de l'assistance maternelle - à travers les 200 ans passés), , Igaku shoin 医学書院, Tōkyō, p. 110-135.

TAmai Kingo 玉井金五 \& Sugita Naho, 2015, « Nihon ni okeru jinkō no $<$ ryō $><$ shitsu $>$ gainen to shakai seisaku no shiteki tenkai - Ueda Sadajirō kara Minoguchi Tokijirōe 》日本における人口の<量 $><$ 質 $>$ 概念と社会 政策の史的展開一上田貞次郎から美濃口時次郎 - (Le développement historique des concepts de <quantité> et de <qualité> en politique sociale au Japon : d'Ueda Sadajirō à Minoguchi Tokijirō), Keizaigaku zasshi 経済学雑 誌 (Revue en économie), vol. 3, $\mathrm{n}^{\circ}$ 1, septembre, p. 25-40. 
The United Nations General Assembly, 1961, "Resolution 1710 (XVI). United Nations Development Decade A Programme for International Economic Co-Operation (I)16 of 19 December 1961. 1084th Plenary Meeting”.

Tipton Elise K., 1994, "Birth Control and the Population Problem in Prewar and Wartime Japan", Japanese Studies 14, nº 1, p. 54-64.

Tipton Elise K., 1997, "Birth Control and the Population Problem", in Elise K. Tipton (ed.) Society and the State in Interwar Japan, Routledge, London and New York, p. 42-62.

Uchino Sumiko, 1966, "Comparative Study of Tohoku and Kyushu Region Observed from Demographic Characteristics and Life Behavior", Annual Reports of the Institute of Population Problems English Summary, $\mathrm{n}^{\circ} 11$, p. 81-82.

WATANABE Jō 渡辺定, 1960, « Jinkō no shitsu no genjō to mondaiten »人口 の質の現状と問題点 (La qualité actuelle de la population et les problèmes), in JINKŌ MONDAI SHINGIKAI, Jinkō mondai shingikai dainijukkai sōkai giji sokkiroku 人口問題審議会第二十回総会議事速記録 (Minutes de la $20^{\mathrm{e}}$ réunion générale du Conseil consultatif général sur les problèmes de population), 25 mars.

Weiner, Michael A., 1994, Race and Migration in Imperial Japan, Sheffield Centre for Japanese Studies/Routledge Series, Routledge, London.

Yamazaki Kiyoko 山崎喜代子 (dir.), 2013, Seimei no rinri : Yūsei seisaku no keifu 生命の倫理 優生政策の系譜 (L'éthique de la vie : la généalogie des politiques eugénistes), Kyūshū daigaku shuppankai 九州大学出版会, Fukuoka.

ZAIDAN HŌJIN JINKŌ MONDAI KENKYŪKAI 財団法人人口問題研究会 (Fondation pour la recherche en matière de problèmes de population), 1962, 
《Jinkō shishitsu kōjō ni kansuru taisaku yōkō ketsugi »人口資質向上 に関する対策要綱決議 (Résolution concernant les mesures relatives à la promotion de la qualité de la population), 8 mai.

Résumé : Afin d'analyser les mesures politiques d'amélioration de la qualité de la population dans l'après-guerre au Japon, le présent article contextualise les discours tenus autour de la « qualité de la population 》 (jinkō shishitsu人口資 質, plus largement jinkō no shitsu 人口の質) qui soutinrent les tentatives de l'État japonais dans sa gouvernance de la population depuis l'avant-guerre. D'un côté, la continuité historique du concept de qualité de la population permet de situer les mesures d'après-guerre sur le prolongement des différentes tentatives entreprises dès l'avant-guerre pour promouvoir la race japonaise. De l'autre côté, les politiques d'après-guerre partent de la redéfinition de la notion de « développement » (kaihatsu 開発) qui ne manqua pas de s'inviter dans les débats. Cette perspective nous incite à dépasser l'histoire de l'eugénisme et à proposer un cadre plus large de l'histoire des politiques de population dans le Japon moderne.

Mots-clés: qualité de la population, politiques de population, politique sociale, eugénisme, euthénisme, ethnie, développement

Abstract: This paper examines the policy proposal in postwar Japan that aimed at improving population quality. In doing so, the paper adopts a longue durée perspective, and contextualizes the policy proposal in terms of the discourses on “population quality” (jinkō shishitsu 人口資質, and to a larger extent, jinkō no shitsu 人口の質) that long underpinned the Japanese State's attempts to govern its population from the prewar period onward. The paper, on the one hand, brings to the fore elements of historical continuity observed in the deployment of the notion of population quality in state policies, thus showing that the postwar proposal to improve population quality was a culmination of various attempts from the prewar period to improve the Japanese race. On the other hand, it also argues that the postwar policy proposal departed from its prewar counterpart, partly because the redefined notion of "development" (kaihatsu 開発) additionally penetrated the population debate. While this case study urges us to look beyond the history of engenics, it also aims at 
offering a more expansive framework to appreciate a history of population policies in modern Japan.

Keywords: Population quality, population policy, socialpolicy, engenics, euthenics, minzoku, kaihatsu

キーワード：人口資質、人口政策、社会政策、優生学、優境学、 民族、開発 